\title{
Experimental social rejection increases dyspnoea perception and neural processing of respiratory sensations in healthy subjects
}

\author{
To the Editor:
}

Patients suffering from dyspnoea frequently report social rejection as a severe limitation of their lives [1]. While the effects of different social challenges on the respiratory system are already established in animal models [2, 3], little is known about potential consequences of social rejection on the perception and neural processing of dyspnoea in humans. Detrimental effects of social rejection are well documented for other aversive somatic symptoms $[4,5]$. The current study examined whether social rejection would increase perceived dyspnoea and whether this would be paralleled by increased neural processing of respiratory sensations as measured with respiratory-related evoked potentials (RREPs) in the electroencephalogram (EEG).

39 healthy females (median (range) age 19.00 (18.00-41.00) years) took part in this study after providing written consent (ethical approval by the University of Leuven ethics committee number G-2016-10-646). Each participant underwent a rejection, inclusion and control condition in a counterbalanced order (figure 1a). The rejection and inclusion conditions were created using the well-validated and clinically reliable [7, 8] Cyberball paradigm [8] (figure 1b). Cyberball is a virtual ball-tossing game in which participants believe they are playing a ball game with two other real players. In reality, no other players exist and participants are playing a computer-generated game. During inclusion, participants receive the ball for $\sim 33 \%$ of the game while during rejection participants receive the ball only for $\sim 6 \%$. The effectiveness of the Cyberball manipulation was assessed by three ratings [8] on feeling accepted-rejected (1: accepted; 9: rejected), received ball throws (0-100\%) and a mood scale with lower values representing more negative mood. The control condition, which served as a baseline including no social component, was created using another virtual ball-tossing game that explicitly stated that no other players are involved (figure 1c). Each condition consisted of two consecutive blocks containing 30 ball throws each ( $\sim 3 \mathrm{~min}$ ).

Each block was followed by a 3-min dyspnoea phase during which an individually tailored strong level of dyspnoea was induced by inspiratory resistive loads. This dyspnoea level was individually predetermined prior to the experimental phase by presenting different inspiratory resistive load magnitudes repeatedly until a subjective level of strong dyspnoea intensity was reached ( 5 on the modified Borg scale; mean $+\mathrm{SD}$ $\left.27.41+13.23 \mathrm{cmH}_{2} \mathrm{O} \cdot \mathrm{L}^{-1} \cdot \mathrm{s}^{-1}\right)$. Simultaneously, in the dyspnoea phase, RREPs were evoked by applying inspiratory occlusions $(150 \mathrm{~ms})$ randomly to every second to fifth inspiration while EEG was recorded continuously. During the dyspnoea phase, participants were breathing through a breathing circuit via a mouthpiece while wearing a nose-clip. The circuit consisted of a two-way nonrebreathing valve with the inspiratory port connected to a pneumotachograph and a loading manifold, to administer the inspiratory resistive loads (all Hans Rudolph Inc., Shawnee, KS, USA), and an occlusion device (Aspire Products, Gainesville, FL, USA) used to trigger the inspiratory occlusions [9]. Respiratory variables including breathing frequency $(f)$, inspiratory time $(t \mathrm{I})$, tidal volume $(V \mathrm{~T})$, mean airflow $\left(V^{\prime}\right)$ and peak inspiratory mouth pressure (PImax) were calculated based on the continuously recorded mouth pressure and airflow signals. After each dyspnoea phase ratings of dyspnoea intensity, unpleasantness and threat were obtained using visual analogue scales (0: not noticeable/not unpleasant/not threatening; 100: maximally imaginable intensity/unpleasantness/threat).

@ERSpublications

Social rejection is associated with increased dyspnoea perception and neural processing of respiratory sensations http://ow.ly/p5hA30mcZMS

Cite this article as: Herzog M, Sucec J, Vukovic M, et al. Experimental social rejection increases dyspnoea perception and neural processing of respiratory sensations in healthy subjects. Eur Respir J 2019; 53: 1801409 [https://doi.org/10.1183/13993003.01409-2018]. 
a)

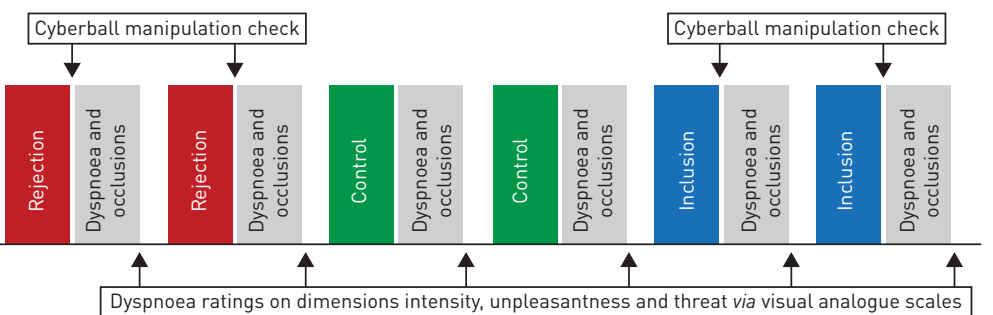

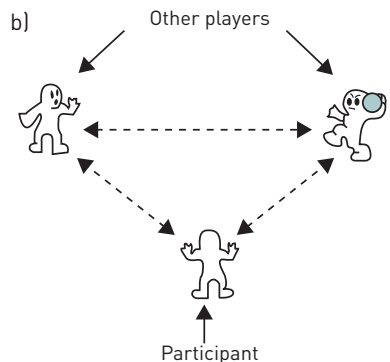

c)

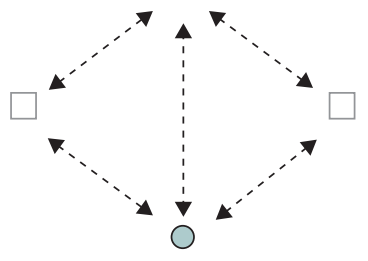

d) 10

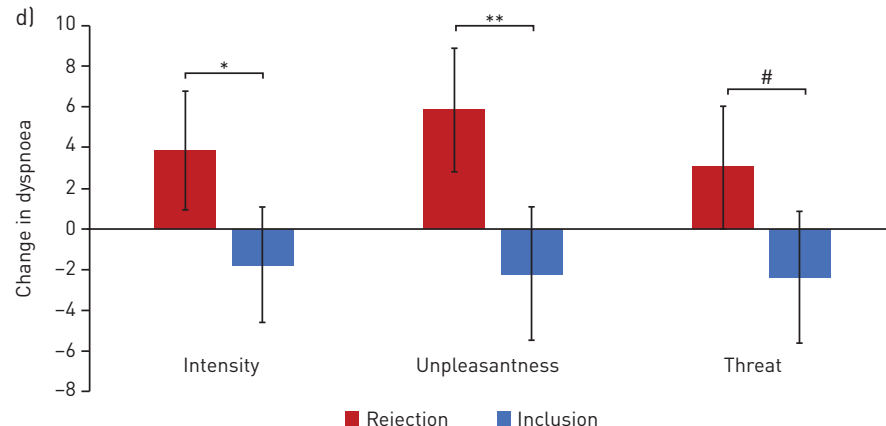

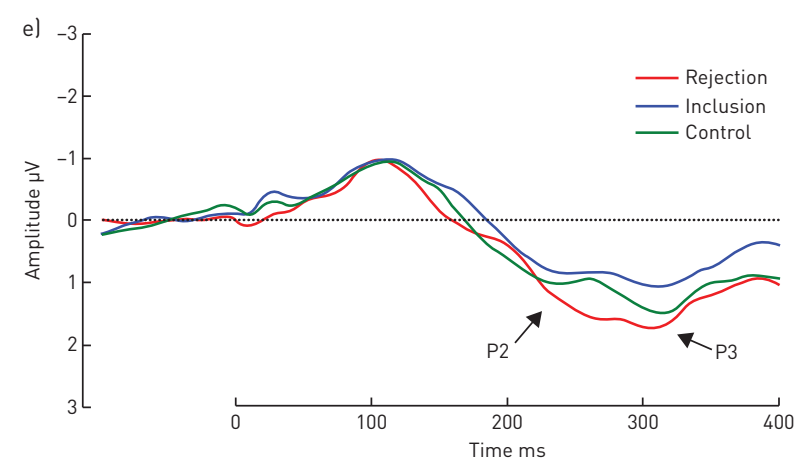

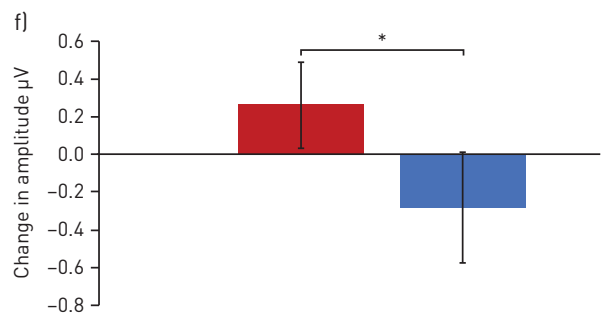

P2

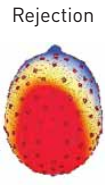

P3

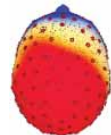

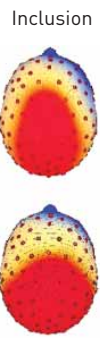
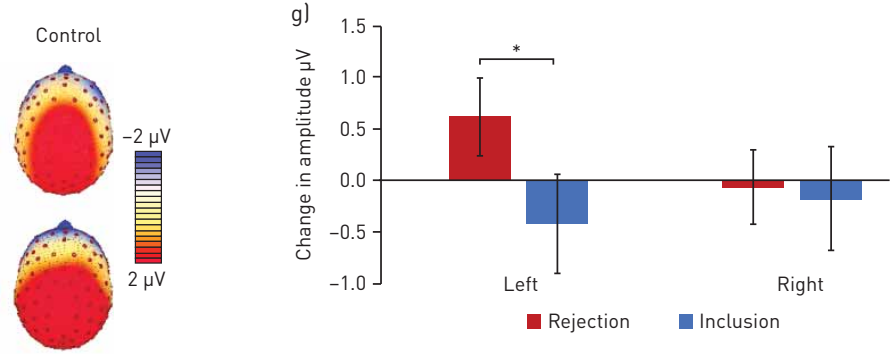

FIGURE 1 a) Schematic representation of the experimental phase for one exemplary participant. The Cyberball manipulation check included ratings on feeling accepted-rejected (1: accepted; 9: rejected), received ball throws from other players (0-100\%) and a mood scale with lower values representing more negative mood. b) Schematic representation of the Cyberball game used to create the rejection and inclusion condition. Dotted arrows indicate all possible trajectories of the ball. c) Schematic representation of the ball-tossing game used to create the control condition. During this game, participants observed a ball moving vertically up/down the screen. Whenever the ball diverted from this trajectory and landed in a left/right square, participants had to indicate this via button press. Dotted arrows indicate all possible trajectories of the ball. d) Mean $\pm S E$ change in dyspnoea ratings including intensity, unpleasantness and threat for the rejection ( $\Delta$ rejection=rejection-control) compared to the inclusion condition (Ainclusion=inclusion-control). Positive values indicate higher and negative values lower dyspnoea intensity, unpleasantness and threat. e) Grand average waveform $(\mu \mathrm{V})$ of the respiratory-related evoked potential (RREP) for the rejection, inclusion and control condition with corresponding P2 and P3 topographies. Data were processed offline using BESA Research 6.0 (BESA GmbH, Gräfelfing, Germany). Data were filtered (high-pass: $0.1 \mathrm{~Hz}$; low-pass: $30 \mathrm{~Hz}$; notch: $50 \mathrm{~Hz}$ ), artefact corrected and average re-referenced. Epochs $200 \mathrm{~ms}$ before and $1000 \mathrm{~ms}$ after occlusion onset were extracted and averaged. Based on previous research [6], P2 and P3 were identified as positive subject-specific peaks in the latency around 160-230 ms after occlusion onset for the P2 and in the latency around 250-350 ms after occlusion onset for the P3. Mean amplitudes for P2 and P3 were then calculated by averaging the signal in a latency window around these subject-specific peaks (P2: \pm 20 ms; P3: \pm 30 ms) across the respective electrodes (P2-left hemisphere: 29/30/36; P2-right hemisphere: 104/105/111; P3-left hemisphere: 7/30/31; P3-right hemisphere: 80/105/106). Mean \pm SE change in mean amplitude ( $\mu \mathrm{V})$ of f) P2 and g) P3 for the rejection ( $\Delta$ rejection=rejection-control) compared to the inclusion condition ( $\Delta$ inclusion=inclusion-control) across both brain hemispheres ( $f$ ) and for left/ right hemispheres separately (g). No significant differences were found for the earlier RREP components Nf, P1 and N1, which represent more sensory first-order neural processing of respiratory sensations [6] (all $p>0.063$ ). ${ }^{*}: p<0.05 ;{ }^{* *}: p<0.01 ;{ }^{\#}: p=0.07$. 
129-channel EEG (Philips EGI, Eugene, OR, USA) was recorded at $250 \mathrm{~Hz}$ during each dyspnoea phase (reference: Cz). The later RREP components P2 and P3 (>150 ms post-occlusion) were analysed as they represent cognitive higher-order neural processing of respiratory sensations [6] and are susceptible to social modulations.

Statistical analyses were conducted using SPSS 24 (IBM Corp., Armonk, NY, USA). Ratings, respiratory variables and P2/P3 amplitudes were averaged across the two blocks of each condition. Ratings of the Cyberball manipulation check were analysed using dependent t-tests/Wilcoxon signed-rank tests. To investigate the pure rejection and inclusion effects on the respiratory variables and the main outcomes of interest (dyspnoea ratings and $\mathrm{P} 2 / \mathrm{P} 3$ ), difference scores were calculated for the rejection $(\Delta$ rejection=rejection-control) and inclusion condition ( $\Delta$ inclusion=inclusion-control). Respiratory variables and dyspnoea ratings were compared with dependent t-tests/Wilcoxon signed-rank tests ( $\Delta$ rejection versus $\Delta$ inclusion). P2 and P3 mean amplitudes were analysed via ANOVAs with the within-factors condition ( $\Delta$ rejection/ $\Delta$ inclusion) and brain hemisphere (left/right). Interaction effects were followed up using dependent t-tests ( $\Delta$ rejection versus $\Delta$ inclusion per hemisphere). The significance level was $\mathrm{p}<0.05$ unless corrected for multiple comparisons using the modified false discovery rate [10].

All ratings of the Cyberball manipulation check (corrected: $\mathrm{p}<0.024)$ differed significantly (all $\mathrm{p}<0.001$ ) confirming a successful induction of social rejection and inclusion. For rejection, higher rejection (median (range) rejection: 7.50 (3.00-8.00); mean \pm SD inclusion 3.22 \pm 1.57 ), fewer ball throws (mean \pm SD rejection 7.28 \pm 3.84 ; median (range) inclusion 31.00 (18.50-55.00)) and more negative mood (mean $\pm \mathrm{SD}$ rejection $5.01 \pm 1.21$; mean \pm SD inclusion $5.91 \pm 1.05$ ) were reported.

No difference between $\Delta$ rejection and $\Delta$ inclusion was found for any respiratory variable (all $\mathrm{p}>0.20$ ). The respective values for rejection, inclusion and control conditions were for $f$, mean \pm SD $13.01 \pm 4.32,13.10 \pm 5.40$ and $12.62 \pm 4.66$ breaths per min; for $t \mathrm{I}$, mean $\pm \mathrm{SD} 2.80 \pm 0.99,2.94 \pm 1.14$ and $2.98 \pm 1.21 \mathrm{~s}$; for $V \mathrm{~T}$, median (range) $0.54(0.36-1.29), 0.58(0.36-1.22)$ and $0.58(0.33-1.27) \mathrm{L}$; for $V^{\prime}$, mean \pm SD $0.22 \pm 0.07,0.22 \pm 0.06$ and $0.22 \pm 0.07 \mathrm{~L} \cdot \mathrm{s}^{-1}$; and for PImax, mean \pm SD $-10.33 \pm 4.72,-10.30 \pm 4.90$ and $-10.23 \pm 4.78 \mathrm{cmH}_{2} \mathrm{O}$.

A significant difference (corrected: $\mathrm{p}<0.027$ ) was found between $\Delta$ rejection and $\Delta$ inclusion conditions for dyspnoea intensity $(p=0.01)$ and unpleasantness $(p=0.005)$ with higher ratings for $\Delta$ rejection compared to $\Delta$ inclusion. A similar trend was found for dyspnoea threat $(p=0.07)$ (figure $1 d)$. Overall, the dyspnoea ratings confirm a successful dyspnoea induction for rejection, inclusion and control conditions with respective mean \pm SD values for intensity, $52.67+19.04,47.05 \pm 17.44$ and $48.82 \pm 19.81$; unpleasantness, 55.60 $\pm 21.45,47.54 \pm 19.55$ and $49.74 \pm 20.17$; and threat, $37.28 \pm 25.06,31.88 \pm 20.55$ and $34.27 \pm 24.43$.

For the P2, a significant main effect was found for the factor condition $(p=0.04)$ with higher mean amplitudes for $\Delta$ rejection compared to $\Delta$ inclusion. For the P3, a significant interaction effect for the factor condition and hemisphere was found $(p=0.01)$. The follow-up tests (corrected: $p<0.033$ ) revealed a significant difference between both conditions for the left $(p=0.016)$, but not the right hemisphere $(\mathrm{p}=0.80)$ with larger mean amplitudes for $\Delta$ rejection compared to $\Delta$ inclusion (figure $1 \mathrm{e}-\mathrm{g}$ ).

The present findings demonstrate increased dyspnoea intensity and unpleasantness after social rejection compared to inclusion with a similar trend for dyspnoea threat. Consistent with the ratings, larger amplitudes for RREP components P2 and P3 were found after rejection compared to inclusion while respiratory variables showed no differences. Together, the current findings indicate that social rejection increases perceived dyspnoea and the neural processing of respiratory sensations in the absence of respiratory changes.

These findings are in line with previous studies demonstrating aversive effects of rejection on breathing in animals $[2,3]$ and on other somatic symptoms in humans [4, 5]. For example, Bernstein and Claypool [4] showed higher pressure pain sensitivity after rejection compared to inclusion using the same Cyberball paradigm. Similarly, CANAIPA et al. [5] found increased electrical pain reports after rejection compared to inclusion. Notably, overlapping brain areas including the insula and dorsal anterior cingulate cortex are activated during the experience of rejection [11] and dyspnoea [12]. This might suggest a potential underlying brain mechanism by which rejection could potentiate the perception of dyspnoea. However, literature also exists that challenges the prediction of overlapping brain areas [13]. Therefore, future neuroimaging studies are required to further investigate this hypothesis and to substantiate potential hemispherical differences herein.

The current findings might have an important clinical implication. Social rejection is a severe problem in dyspnoeic patients [1] and might be a contributing factor to the worsening of dyspnoea. However, dyspnoea and the resulting social rejection are frequently overlooked by healthcare professionals, which might further contribute to its aggravation [14]. Therefore, social rejection should be recognised by clinicians and specifically targeted in the treatment of dyspnoeic patients to break the vicious cycle of 
dyspnoea-social rejection-dyspnoea. For example, a cost-effective strategy could be Internet-based technologies to implement online group activities/support into the lives of dyspnoeic patients to reduce their social rejection [15].

Certain limitations of this study are noteworthy. This study was conducted in healthy females who were mainly psychology students. This limits the generalisation to dyspnoeic patient and male populations, particularly because females might react more strongly to social rejection than males [16]. Additionally, the ratings of the Cyberball manipulation check might have in itself potentiated the social rejection/inclusion manipulation. Furthermore, additional dyspnoea induction methods (e.g. carbon dioxide inhalation, exercise-induced dyspnoea and vicarious dyspnoea [17]) should be applied to allow the investigation of other dyspnoea qualities (e.g. chest tightness or air hunger). The present study only investigated the effects of brief social rejection. Thus, future studies should investigate the effects of repeated or long-term social rejection.

Michaela Herzog, Josef Sucec, Milica Vukovic, Ilse Van Diest, Omer Van den Bergh and Andreas von Leupoldt $\odot$ Health Psychology, University of Leuven, Leuven, Belgium.

Correspondence: Michaela Herzog, Research Group Health Psychology, University of Leuven, Tiensestraat 102, 3000 Leuven, Belgium. E-mail: michaela.herzog@kuleuven.be

Received: July 252018 | Accepted after revision: Oct 072018

Author contributions: A. von Leupoldt, O. Van den Bergh, I. Van Diest, M. Vukovic, J. Sucec and M. Herzog conceptualised the research design and research protocol. M. Herzog and M. Vukovic were responsible for the data collection. M. Herzog and A. von Leupoldt were responsible for the statistical analysis. M. Herzog and A. von Leupoldt had access to all the data in the study and take responsibility for the integrity of the data as well as the data analysis. M. Herzog created the figure. M. Herzog wrote the manuscript. M. Herzog, J. Sucec, M. Vukovic, I. Van Diest, O. Van den Bergh and A. von Leupoldt provided feedback on the protocol, interpretation of the study results and on the manuscript. All authors read and approved the final version.

Conflict of interest: M. Herzog has nothing to disclose. J. Sucec has nothing to disclose. M. Vukovic has nothing to disclose. I. Van Diest has nothing to disclose. O. Van den Bergh has nothing to disclose. A. von Leupoldt reports receiving grants from Research Fund KU Leuven, Herculesstichting and Methusalem during the conduct of the study.

Support statement: This study was supported by grants from the Research Fund KU Leuven, Belgium (STRT/13/002 and DBOF/14/021), by an infrastructure grant from the Herculesstichting, Belgium (AKUL/13/07), and by the "Asthenes" long-term structural funding Methusalem grant (METH/15/011) by the Flemish Government, Belgium. The funders had no role in the study design, data collection, data analysis, data interpretation or writing of the manuscript. M. Herzog and A. von Leupoldt had full access to all data. All authors had the final responsibility for the decision to submit the manuscript for publication. Funding information for this article has been deposited with the Crossref Funder Registry.

\section{References}

1 Hutchinson A, Barclay-Klingle N, Galvin K, et al. Living with breathlessness: a systematic literature review and qualitative synthesis. Eur Respir J 2018; 51: 1701477.

2 Brouillard C, Carrive P, Camus F, et al. Long-lasting bradypnea induced by repeated social defeat. Integr Comp Physiol 2016; 311: R352-R364.

3 Battaglia M, Ogliari A, D'Amato F, et al. Early-life risk factors for panic and separation anxiety disorder: insights and outstanding questions arising from human and animal studies of $\mathrm{CO}_{2}$ sensitivity. Neurosci Biobehav Rev 2014; 46: 455-464.

4 Bernstein MJ, Claypool HM. Social exclusion and pain sensitivity: why exclusion sometimes hurts and sometimes numbs. Pers Soc Psychol Bull 2012; 38: 185-196.

5 Canaipa R, Treister R, Lang M, et al. Feeling hurt: pain sensitivity is correlated with and modulated by social distress. Clin J Pain 2016; 32: 14-19.

6 Chan P-YS, Davenport PW. Respiratory related evoked potential measures of cerebral cortical respiratory information processing. Biol Psychol 2010; 84: 4-12.

7 Hartgerink $\mathrm{CH}$, van Beest I, Wicherts JM, et al. The ordinal effects of ostracism: a meta-analysis of 120 Cyberball studies. PLoS One 2015; 10: e0127002.

8 Williams KD, Cheung CK, Choi W. Cyberostracism: effects of being ignored over the Internet. J Pers Soc Psychol 2000; 79: 748-762.

9 Herzog M, Sucec J, Van Diest I, et al. Reduced neural gating of respiratory sensations is associated with increased dyspnoea perception. Eur Respir J 2018; 52: 1800559.

10 Benjamini Y, Yekutieli D. The control of the false discovery rate in multiple testing under dependency. Ann Stat 2001; 29: 1165-1188.

11 Eisenberger NI. Does rejection hurt? An fMRI study of social exclusion. Science 2003; 302: 290-292.

12 Herigstad M, Hayen A, Wiech K, et al. Dyspnoea and the brain. Respir Med 2011; 105: 809-817.

13 Woo C-W, Koban L, Kross E, et al. Separate neural representations for physical pain and social rejection. Nat Commun 2014; 5: 5380.

14 Gysels M, Higginson IJ. Access to services for patients with chronic obstructive pulmonary disease: the invisibility of breathlessness. J Pain Symptom Manage 2008; 36: 451-460. 
15 Chen Y-RR, Schulz PJ. The effect of information communication technology interventions on reducing social isolation in the elderly: a systematic review. J Med Internet Res 2016; 18: e18.

16 Benenson JF, Markovits H, Hultgren B, et al. Social exclusion: more important to human females than males. PLoS One 2013; 8: e55851.

17 Herzog M, Sucec J, Van Diest I, et al. Observing dyspnoea in others elicits dyspnoea, negative affect, and brain responses. Eur Respir J 2018; 51: 1702682.

Copyright @ERS 2019 